\title{
Research Article \\ EFFECT OF AREA SPECIFIC MINERAL MIXTURE ON SERUM BIOCHEMICAL PARAMETERS AND MILK YIELD IN DAIRY CATTLE
}

\author{
PANDEY V.K., GENDLEY M.K. ${ }^{*}$, TIWARI S.P. AND PRUSTY SONALI \\ College of Veterinary Science and Animal Husbandry, Anjora, Durg, 491001, Chhattisgarh Kamdhenu Vishwavidyalaya, Raipur, 492006, Chhattisgarh \\ *Corresponding Author: Email-drgmanoj.ann@gmail.com
}

Received: December 12, 2017; Revised: December 27, 2017; Accepted: December 28, 2017; Published: January 15, 2018

\begin{abstract}
In the present study effect of area specific mineral mixture on the serum enzymes viz. alanine transaminase (ALT); aspartate transaminase (AST) and alkaline Phosphatase (ALP), biochemical parameters viz. serum total protein, albumin, globulin and cholesterol concentration of cattle at three agro-climatic zones (plateau, plain and hills) of Chhattisgarh state were studied. Fifteen dairy cows in each zone were randomly distributed into three groups viz. negative control- natural grazing without mineral supplementation; control group - common mineral mixture supplemented @ 80 gm/day/animal along with natural grazing and treatment grouparea specific mineral mixture (80 gm/day/animal) supplemented along with natural grazing. The serum albumin and cholesterol levels were non-significantly different among the groups except total protein, globulin, ALT and milk yield. The serum total protein values were significantly $(p<0.05)$ higher in both the supplemented groups in all the agro-climatic zones, however value of globulin was significantly higher in plain zone, milk yield was significantly higher in plateau zone and ALP value was significantly $(p<0.05)$ higher in plateau and plain zones with commercial and area specific mineral supplemented groups than negative control group. It may be concluded that the effect of commercial and area specific mineral mixture revealed similar effect on serum enzymes, biochemical parameters and milk yield.

Key words- Agro-climatic zones, Area specific mineral mixture, Serum enzymes, Serum protein.
\end{abstract}

Citation: Pandey V. K., et al., (2018) Effect of Area Specific Mineral Mixture on Serum Biochemical Parameters and Milk Yield in Dairy Cattle. International Journal of Agriculture Sciences, ISSN: 0975-3710 \& E-ISSN: 0975-9107, Volume 10, Issue 1, pp.-4965-4968. DOl: http://dx.doi.org/10.9735/0975-3710.10.1.4965-4968

Copyright: Copyright@2018 Pandey V. K., et al., This is an open-access article distributed under the terms of the Creative Commons Attribution License, which permits unrestricted use, distribution and reproduction in any medium, provided the original author and source are credited.

Academic Editor / Reviewer: Dr Jasmine Rani K, Mathi P. Madhu, Dr Sheikh Bilal Ahmad, Chandra Gulab, Padodara R.J., Dutta Narayan, Dr Ankit Kumar, Dhok A.P., Saidj D., Swelum A.

\section{Introduction}

Chhattisgarh is Central Indian state with tropical climate and paddy is the principal crop with other major crops as coarse grains, wheat, maize, groundnut, pulses and oilseeds. Agro-climatically, Chhattisgarh may be divided into 3 distinct zones viz. plain, plateau and northern hills. The varying agro climatic conditions, topography and soil type of Chhattisgarh are responsible for varying mineral composition of feed/fodder plants of different regions.

Nature of soil like $\mathrm{pH}$, salinity, alkalization and organic matter content, season or climate, agricultural practices contribute to the effects of soil on plant mineral content [1], adjusting the availability of minerals to livestock and reflecting the production performance of animals. So, the mineral status of soil does not necessarily indicate either the rate of mineral uptake by plants or their subsequent intake and utilization by animals [2].

Mineral deficiency in livestock animals is responsible for poor growth, deficiency disease, decreased milk yield, reproductive disorders, and decreased immunity. During the past decade, significant advances have been made in understanding the effects of trace-element supplements on the milk production of dairy cows [3]. Data regarding the role of mineral supplementation affecting milk yield performance in cattle is scant. Supplementation of all the elements in diet may not be desirable always, because many of them such as $\mathrm{Mg}, \mathrm{S}, \mathrm{K}, \mathrm{I}, \mathrm{Co}$, Fe and $\mathrm{Mn}$ are present in required concentrations in feeds and fodders of specific areas. Significant changes in major physiological and biochemical parameters have been observed due to deficiencies of minerals like $\mathrm{Ca}, \mathrm{Cu}$ and $\mathrm{Zn}$. [4]. So, the present study was planned to evaluate the effect of area specific mineral mixtures on serum constituents in different agro climatic zones of Chhattisgarh.

\section{Materials and Methods \\ Animal Management and Feeding}

An experiment was conducted to evaluate effect of area specific mineral mixture on serum biochemical and milk yield status of dairy cattle in three agro-climatic zones viz. northern hill, plateau, and plain regions of Chhattisgarh state. Fifteen dairy cattle of mid phase of second lactation from hill, plateau and plain regions were selected for experiment from Semra, Redai and Atra villages of Surguja, Kanker and Dhamtari districts. Cows from each agro climatic zone were randomly divided into three groups namely negative control, control and treatment with five cows in each. Animals were managed in semi intensive condition. Well ventilated cow shed with facilities for individual feeding was provided. Animals were allowed for voluntary grazing on available pasture grass for at least four hours in the morning and two hours in the evening. The feedstuffs commonly available in all the zones were wheat straw, paddy straw and dub grass. Radium blue for negative control, red for control and green for treatment group was painted over the horn and pole region for identification of the animals. All animals were sprayed with Butox ( $5 \mathrm{ml} /$ litre) one week before start of experiment to control the ectoparasites. Deworming was done with oral administration of $2.5 \%$ w/v albendazole suspension at the rate of $7.5 \mathrm{mg} / \mathrm{kg} \mathrm{BW}$. Duration of each experiment was 90 days. The details of experimental design are presented in [Table-1]. Negative control animals were grazed without mineral supplementation while animals of control group were supplemented @ 80 gm/day/animal with commercial mineral mixture along with natural grazing. The ingredients composition of commercially available mineral mixture (common) is provided in [Table-2]. Animals of treatment group were supplemented @ $80 \mathrm{gm} /$ day/animal with area specific mineral mixture along with natural grazing. Different agro-climatic zone wise area specific mineral mixture was prepared by the Department of Animal Nutrition, College of 
Veterinary Science and Animal Husbandry, Anjora, Durg, Chhattisgarh, under the project of Rashtriya Krishi Vikas Yojna (RKVY). The ingredients compositions of area specific mineral mixtures are provided in [Table-3].

\begin{tabular}{|c|c|c|c|c|c|c|c|c|c|}
\hline \multicolumn{5}{|c|}{ 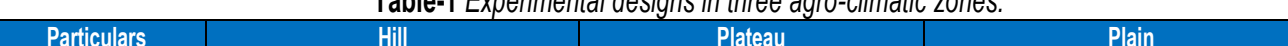 } & Plateau & & \multirow{2}{*}{\multicolumn{3}{|c|}{$\begin{array}{c}\text { Plain } \\
15\end{array}$}} \\
\hline № of animal & \multicolumn{3}{|c|}{15} & \multicolumn{3}{|c|}{15} & & & \\
\hline Treatment & NC & C & $\mathrm{T}$ & NC & $C$ & $T$ & $\mathrm{NC}$ & $C$ & $T$ \\
\hline No of animal & 5 & 5 & 5 & 5 & 5 & 5 & 5 & 5 & 5 \\
\hline $\begin{array}{l}\text { Commercial Mineral } \\
\text { Mix. (g/day/animal) }\end{array}$ & - & 80 & - & - & 80 & - & - & 80 & - \\
\hline $\begin{array}{l}\text { Area specific Mineral } \\
\text { Mix. (g/day/animal) }\end{array}$ & - & - & 80 & - & - & 80 & - & - & 80 \\
\hline Feeding by & Grazing & Grazing & Grazing & Grazing & Grazing & Grazing & Grazing & Grazing & Grazing \\
\hline
\end{tabular}

\begin{tabular}{|c|c|c|c|c|c|c|c|c|c|c|c|c|c|c|c|}
\hline \multicolumn{10}{c|}{ Table-2 Composition of commercial mineral mixture utilized in the experiments for the three agro-climatic zones } \\
\hline Particulars & $\begin{array}{c}\text { Moisture } \\
* *\end{array}$ & $\begin{array}{c}\text { Ca } \\
*\end{array}$ & $\begin{array}{c}\text { P } \\
*\end{array}$ & $\begin{array}{c}\text { Mg } \\
*\end{array}$ & $\begin{array}{c}\text { S } \\
*\end{array}$ & $\begin{array}{c}\text { Salt } \\
\text { mix. } \\
*\end{array}$ & $\begin{array}{c}\text { Zn } \\
*\end{array}$ & $\begin{array}{c}\text { Fe } \\
*\end{array}$ & $\begin{array}{c}\text { I } \\
*\end{array}$ & $\begin{array}{c}\text { Cu } \\
*\end{array}$ & $\begin{array}{c}\text { Co } \\
*\end{array}$ & $\begin{array}{c}\text { F } \\
* *\end{array}$ & TA & AIA \\
\hline $\begin{array}{c}\text { Mineral } \\
\text { inclusion level }\end{array}$ & 5.00 & 16.00 & 9.00 & 4.00 & 1.40 & 22.00 & 0.30 & 0.30 & 0.02 & 0.08 & 0.10 & 0.01 & 0.05 & $75-82$ & 3 \\
\hline
\end{tabular}

Table-3 Composition of area specific mineral mixture utilized in the experiment for the three agro-climatic zones
\begin{tabular}{|c|c|c|c|c|c|c|c|c|c|}
\hline Region & $\begin{array}{c}\mathrm{Ca} \\
(\%)\end{array}$ & $\begin{array}{c}\mathrm{P} \\
(\%)\end{array}$ & $\begin{array}{c}\mathrm{Mg} \\
(\mathrm{g})\end{array}$ & $\begin{array}{c}\mathrm{Na} \\
(\mathrm{mg})\end{array}$ & $\mathrm{K}(\mathrm{mg})$ & $\begin{array}{c}\mathrm{Cu} \\
\mathrm{mg})\end{array}$ & $\begin{array}{c}\mathrm{Fe} \\
(\mathrm{mg})\end{array}$ & $\begin{array}{c}\mathrm{Zn} \\
(\mathrm{mg})\end{array}$ & $\begin{array}{c}\mathrm{Mn} \\
(\mathrm{mg})\end{array}$ \\
\hline Hill & 25.50 & 12.75 & 6.00 & 5.9 & 100 & 1200 & 1500 & 9600 & 1500 \\
\hline Plateau & 22.00 & 11.00 & 4.50 & 5.9 & 100 & 600 & 1500 & 4800 & 800 \\
\hline Plain & 18.00 & 9.00 & 3.00 & 5.9 & 100 & 300 & 1500 & 3200 & 1000 \\
\hline
\end{tabular}

\section{Sample collection and preparation}

The blood samples from the animals were collected at 0,45 , and 90 days of experiment. About $9 \mathrm{ml}$ of blood was collected aseptically from jugular vein in a sterilized and vacumized test tube (vacuette) without anticoagulant. The test tubes containing blood were kept in slanting position at room temperature without disturbing up to 3-4 hrs, the clot was broken with the help of pasteur pipette. The serum was centrifuged and stored at $4^{\circ} \mathrm{C}$ in plastic vials for further analysis.

\section{Analysis of serum enzyme}

Serum enzymes like alanine transaminase (ALT), aspartate transaminase (AST), alkaline phosphatase (ALP) were quantified by semi-automatic biochemical analyzer using commercial kit (Beacon Diagnostic Pvt. Ltd. - Kabilpore, Navsari). Serum samples were analyzed for total protein, globulin, albumin, and total cholesterol in semi-automated analyzer by using diagnostic kits (Beacon Diagnostic Pvt. Ltd. - Kabilpore, Navsari). Milk yield of cow was measured after suckling of calves for letdown of milk by measuring cylinder.

\section{Statistical analysis}

For interpretation of the results, the data was subjected to one-way ANOVA following completely randomized design for analysis of variance and significance of differences among groups were tested by Snedecor and Cochran, 1994 [5].

\section{Results and Discussion \\ Serum enzymes}

No significant effect was observed on serum Aspartate transaminase (AST) or Alkaline Phosphatase (ALP) concentration in the animals of different agro climatic zones due to supplementation of either commercial or area specific mineral mixture [Table-4]. But significant $(p<0.05)$ difference was observed in serum ALT concentration among the groups. In a similar study Chaudhary et al,2015 [6] observed no change in activities of serum AST, ALT and ALP enzymes in anestrous buffaloes supplemented either on ionic or chelated mineral mixture. Higher serum ALT was observed in control and treatment group compared to negative control in plateau and plain region. The finding is supported by finding of Sharma et. al. 2011 [7] who reported higher ALT, AST and ALP activities in groups of crossbred growing heifers supplemented with specific minerals than control group (without mineral supplementation). They reported dietary supplementation of selective minerals to be beneficial for improved nutrient utilization, weight gain and normal blood biochemical constituents and mineral status in growing crossbred heifers. In the present study AST and ALP concentration was recorded between 94.8- 104.4 IU/L and 201.2-243.2 IU/L, respectively. AST and ALP concentration recorded in shorthorn cow of Canada was 122.6 and $120.6 \mathrm{U} / \mathrm{L}$ Doornenbal, et. al., 1988 [8]. Exton, [9] reported that AST and ALT concentration were significantly less in the mineral deficient lambs than those supplemented specific minerals ( $\mathrm{Ca}$ and $\mathrm{P}$ ) may be due to enhancement in transamination reaction. Kumar et. al., 2005 [10] observed lower serum ALP in specific mineral deficient buffaloes, whereas lowering in serum ALT and AST was not significant.

\section{Total protein, albumin and globulin and cholesterol concentration}

Serum total protein, albumin, globulin and total cholesterol concentrations in different groups at three agro-climatic zones at days 0, 45 and 90 are provided in [Table-5]. The mineral supplementation did not generate significant difference in serum albumin concentration among the groups in different agro-climatic zones. Serum globulin level differed significantly $(p<0.05)$ on 45 days due to mineral supplementation at plain zone, control and treatment group showed higher albumin level compared to negative control group. However, no significant difference was observed in the experiment groups at other agro-climatic zones. There was no significant variation in serum cholesterol level throughout the experimental period at any of the agro-climatic zones. Sharma et al., 2011[7] observed serum protein, albumin, globulin and cholesterol levels of the animals of Western zone of Odisha to be $4.67 \mathrm{~g} / \mathrm{dL}, 2.21 \mathrm{~g} / \mathrm{dL}, 2.46 \mathrm{~g} / \mathrm{dL}$ and $60.04 \mathrm{mg} / \mathrm{dL}$, respectively. The serum total protein, albumin, globulin and cholesterol concentration in cattle ranged from 75.50 to $79.05 \mathrm{~g} / \mathrm{L}, 37.29$ to $38.19 \mathrm{~g} / \mathrm{L}, 37.82$ to $40.86 \mathrm{~g} / \mathrm{L}$ and 3.25 to $3.62 \mathrm{mmo} / \mathrm{L}$ respectively in different areas (urban, peri urban, rural) of Middle Gangetic Plains Sharma et al., 2011 [7] suggested the values to be within normal physiological range for cattle. The present study followed similar trend and the values were within normal range suggesting healthy animal status. 
Table-4 Mean value of serum enzymes (ALT, AST, ALP) in cow supplemented either area specific (T) or commercial mineral mixture (C) in different agro-climatic zones

\begin{tabular}{|c|c|c|c|c|c|c|c|c|c|c|c|c|c|}
\hline \multirow[t]{2}{*}{ Particulars } & \multirow[b]{2}{*}{ Days } & \multicolumn{4}{|c|}{ Hill } & \multicolumn{4}{|c|}{ Plateau } & \multicolumn{4}{|c|}{ Plain } \\
\hline & & NC & C & $\mathrm{T}$ & Sig. & NC & C & $\mathrm{T}$ & Sig. & NC & C & $\mathrm{T}$ & Sig. \\
\hline \multirow[t]{3}{*}{ ALT (IU/L) } & 0 & $\begin{array}{c}25.6 \\
\pm 1.69\end{array}$ & $\begin{array}{c}26 \\
\pm 1.26\end{array}$ & $\begin{array}{c}27.2 \\
\pm 1.69\end{array}$ & NS & $\begin{array}{l}25.40 \\
\pm 0.75\end{array}$ & $\begin{array}{r}27.80 \\
\pm 1.16\end{array}$ & $\begin{array}{l}26.20 \\
\pm 1.39\end{array}$ & NS & $\begin{array}{l}27.60 \\
\pm 1.21\end{array}$ & $\begin{array}{l}27.20 \\
\pm 1.69\end{array}$ & $\begin{array}{l}28.00 \\
\pm 0.84\end{array}$ & NS \\
\hline & 45 & $\begin{array}{c}26 \\
\pm 1.26\end{array}$ & $\begin{array}{c}28 \\
\pm 1.22\end{array}$ & $\begin{array}{c}28.8 \\
\pm 0.97\end{array}$ & NS & $\begin{array}{r}25.60 \\
\pm 1.12\end{array}$ & $\begin{array}{l}29.60 \\
\pm 0.93\end{array}$ & $\begin{array}{l}27.80 \\
\pm 1.16\end{array}$ & NS & $\begin{array}{l}28.00 \\
\pm 0.84\end{array}$ & $\begin{array}{l}30.40 \\
\pm 1.03\end{array}$ & $\begin{array}{l}30.40 \\
\pm 1.03\end{array}$ & NS \\
\hline & 90 & $\begin{array}{c}27.2 \\
\pm 1.69\end{array}$ & $\begin{array}{c}28.8 \\
\pm 0.97\end{array}$ & $\begin{array}{c}30.6 \\
\pm 0.98\end{array}$ & NS & $\begin{array}{r}25.80 \mathrm{~b} \\
\pm 1.46\end{array}$ & $\begin{array}{l}31.60 \mathrm{a} \\
\pm 0.81\end{array}$ & $\begin{array}{c}29.00 \mathrm{a} \\
\pm 1.38\end{array}$ & * & $\begin{array}{l}27.20 \mathrm{~b} \\
\pm 1.69\end{array}$ & $\begin{array}{c}32.40 \mathrm{a} \\
\pm 0.93\end{array}$ & $\begin{array}{l}31.60 \mathrm{a} \\
\pm 1.12\end{array}$ & * \\
\hline \multirow[t]{3}{*}{$\begin{array}{c}\text { AST } \\
\text { (IU/L) }\end{array}$} & 0 & $\begin{array}{r}94.8 \\
\pm 5.04 \\
\end{array}$ & $\begin{array}{c}97 \\
\pm 4.95 \\
\end{array}$ & $\begin{array}{c}96.8 \\
\pm 3.34\end{array}$ & NS & $\begin{array}{r}97.60 \\
\pm 3.57 \\
\end{array}$ & $\begin{array}{r}97.40 \\
\pm 2.18\end{array}$ & $\begin{array}{l}95.60 \\
\pm 2.66\end{array}$ & NS & $\begin{array}{l}94.80 \\
\pm 5.04\end{array}$ & $\begin{array}{l}95.80 \\
\pm 2.85\end{array}$ & $\begin{array}{l}95.60 \\
\pm 2.66\end{array}$ & NS \\
\hline & 45 & $\begin{array}{r}96.8 \\
\pm 3.34\end{array}$ & $\begin{array}{l}101.2 \\
\pm 3.47\end{array}$ & $\begin{array}{r}99.2 \\
\pm 3.69\end{array}$ & NS & $\begin{array}{r}95.60 \\
\pm 2.66\end{array}$ & $\begin{array}{l}101.00 \\
\pm 2.12\end{array}$ & $\begin{array}{r}97.40 \\
\pm 2.18\end{array}$ & NS & $\begin{array}{l}95.60 \\
\pm 2.66\end{array}$ & $\begin{array}{l}99.40 \\
\pm 2.50\end{array}$ & $\begin{array}{l}97.40 \\
\pm 2.18\end{array}$ & NS \\
\hline & 90 & $\begin{array}{c}95.4 \\
\pm 3.04\end{array}$ & $\begin{array}{l}104.4 \\
\pm 4.09\end{array}$ & $\begin{array}{l}103.6 \\
\pm 3.47\end{array}$ & NS & $\begin{array}{l}96.00 \\
\pm 2.39\end{array}$ & $\begin{array}{l}104.20 \\
\pm 2.44\end{array}$ & $\begin{array}{l}102.40 \\
\pm 2.58\end{array}$ & NS & $\begin{array}{l}95.80 \\
\pm 2.85\end{array}$ & $\begin{array}{l}102.40 \\
\pm 2.58\end{array}$ & $\begin{array}{l}102.40 \\
\pm 2.58\end{array}$ & NS \\
\hline \multirow[t]{3}{*}{$\begin{array}{l}\text { ALP } \\
\text { (IU/L) }\end{array}$} & 0 & $\begin{array}{r}201.2 \\
\pm 17.84 \\
\end{array}$ & $\begin{array}{r}207.8 \\
\pm 10.37 \\
\end{array}$ & $\begin{array}{r}205.8 \\
\pm 14.08 \\
\end{array}$ & NS & $\begin{array}{r}223.20 \\
\pm 14.63 \\
\end{array}$ & $\begin{array}{r}226.60 \\
\pm 8.62 \\
\end{array}$ & $\begin{array}{r}223.20 \\
\pm 14.63 \\
\end{array}$ & NS & $\begin{array}{r}220.80 \\
\pm 27.19 \\
\end{array}$ & $\begin{array}{r}226.60 \\
\pm 8.62 \\
\end{array}$ & $\begin{array}{r}223.20 \\
\pm 14.63 \\
\end{array}$ & NS \\
\hline & 45 & $\begin{array}{c}205 \\
\pm 14.72\end{array}$ & $\begin{array}{c}215 \\
\pm 10.69\end{array}$ & $\begin{array}{c}215 \\
\pm 10.69\end{array}$ & NS & $\begin{array}{r}220.80 \\
\pm 27.19 \\
\end{array}$ & $\begin{array}{r}231.40 \\
\pm 12.54\end{array}$ & $\begin{array}{r}226.20 \\
\pm 14.15\end{array}$ & NS & $\begin{array}{r}227.60 \\
\pm 15.37 \\
\end{array}$ & $\begin{array}{r}238.80 \\
\pm 13.17 \\
\end{array}$ & $\begin{array}{r}230.00 \\
\pm 14.31 \\
\end{array}$ & NS \\
\hline & 90 & $\begin{array}{c}205.8 \\
\pm 14.08 \\
\end{array}$ & $\begin{array}{r}222.2 \\
\pm 11.20\end{array}$ & $\begin{array}{c}222.2 \\
\pm 11.20 \\
\end{array}$ & NS & $\begin{array}{l}217.60 \\
\pm 27.06\end{array}$ & $\begin{array}{r}235.80 \\
\pm 12.48 \\
\end{array}$ & $\begin{array}{r}231.40 \\
\pm 12.54 \\
\end{array}$ & NS & $\begin{array}{l}223.20 \\
\pm 14.63\end{array}$ & $\begin{array}{r}243.20 \\
\pm 13.04 \\
\end{array}$ & $\begin{array}{r}238.80 \\
\pm 13.17 \\
\end{array}$ & NS \\
\hline
\end{tabular}

Means in the same row with different superscript $a$ and $b$, are significantly different $(" p<0.05)$ NS= Non-significant

NC - Negative control, C-control, T- treatment, ALT-Alanine transaminase; AST- Aspartate transaminase; ALP- Alkaline Phosphatase

Table-5 Mean value of blood biochemical parameters total protein, albumin, globulin (g/dl) in cow due supplemented either area specific (T) or commercial mineral mixture (C) in different agro-climatic zones

\begin{tabular}{|c|c|c|c|c|c|c|c|c|c|c|c|c|c|}
\hline \multirow[t]{2}{*}{ Particulars } & \multirow[t]{2}{*}{ Days } & \multicolumn{4}{|c|}{ Hill } & \multicolumn{4}{|c|}{ Plateau } & \multicolumn{4}{|c|}{ Plain } \\
\hline & & NC & C & T & Sig & NC & c & T & sig & NC & c & T & Sig. \\
\hline \multirow{3}{*}{$\begin{array}{c}\text { Total protein } \\
(\mathrm{g} / \mathrm{dl})\end{array}$} & 0 & $6.36 \pm 0.15$ & $6.32 \pm 0.14$ & $6.42 \pm 0.19$ & NS & $5.94 \pm 0.17$ & $5.78 \pm 0.17$ & $5.80 \pm 0.16$ & NS & $6.44 \pm 0.19$ & $6.40 \pm 0.19$ & $6.22 \pm 0.18$ & NS \\
\hline & 45 & $6.04^{b} \pm 0.11$ & $6.46^{a} \pm 0.12$ & $6.76 \pm 0.16$ & ** & $5.78^{\circ} \pm 0.17$ & $5.98 \pm 0.17$ & $5.98 \mathrm{a} \pm 0.17$ & & $5.94^{b} \pm 0.17$ & $6.70^{a} \pm 0.13$ & $6.66^{a} \pm 0.14$ & ** \\
\hline & 90 & $6.14^{b} \pm 0.11$ & $6.66^{a b} \pm 0.09$ & $6.78^{a} \pm 0.29$ & ** & $5.80 \pm 0.16$ & $6.18 \pm 0.04$ & $6.12 \pm 0.07$ & NS & $6.26^{b} \pm 0.20$ & $6.94^{a} \pm 0.12$ & $6.74^{a} \pm 0.07$ & ${ }^{* \star}$ \\
\hline \multirow{3}{*}{$\begin{array}{l}\text { Albumin } \\
\text { (g/dl) }\end{array}$} & 0 & $3.02 \pm 0.17$ & $3.00 \pm 0.06$ & $3.04 \pm 0.18$ & NS & $2.94 \pm 0.09$ & $3.10 \pm 0.16$ & $2.96 \pm 0.07$ & NS & $3.04 \pm 0.08$ & $3.10 \pm 0.16$ & $3.06 \pm 0.12$ & NS \\
\hline & 45 & $2.98 \pm 0.10$ & $3.16 \pm 0.05$ & $3.14 \pm 0.21$ & $\overline{N S}$ & $2.96 \pm 0.07$ & $3.30 \pm 0.10$ & $3.20 \pm 0.09$ & NS & $3.06 \pm 0.09$ & $3.28 \pm 0.10$ & $3.20 \pm 0.09$ & NS \\
\hline & 90 & $3.00 \pm 0.06$ & $3.32 \pm 0.02$ & $3.24 \pm 0.22$ & & $2.94 \pm 0.10$ & $3.52 \pm 0.11$ & $3.30 \pm 0.10$ & NS & $3.06 \pm 0.12$ & $3.46 \pm 0.10$ & $3.30 \pm 0.10$ & NS \\
\hline \multirow{3}{*}{$\begin{array}{c}\text { Globulin } \\
\text { (g/dl) }\end{array}$} & 0 & $3.34 \pm 0.21$ & $3.32 \pm 0.15$ & $3.38 \pm 0.22$ & NS & $2.90 \pm 0.20$ & $2.68 \pm 0.18$ & $2.84 \pm 0.20$ & NS & $3.20 \pm 0.13$ & $3.30 \pm 0.24$ & $3.38 \pm 0.12$ & NS \\
\hline & 45 & $3.06 \pm 0.16$ & $3.30 \pm 0.11$ & $3.62 \pm 0.25$ & NS & $2.92 \pm 0.15$ & $2.68 \pm 0.23$ & $2.78 \pm 0.22$ & NS & $2.96^{b} \pm 0.08$ & $3.42^{a} \pm 0.13$ & $3.46^{a} \pm 0.12$ & * \\
\hline & 90 & $3.14 \pm 0.15$ & $3.34 \pm 0.10$ & $3.54 \pm 0.40$ & NS & $2.86 \pm 0.26$ & $2.66 \pm 0.11$ & $2.82 \pm 0.11$ & NS & $3.20 \pm 0.20$ & $3.48 \pm 0.12$ & $3.44 \pm 0.13$ & NS \\
\hline \multirow{3}{*}{$\begin{array}{c}\text { Cholesterol } \\
\text { (g/dl) }\end{array}$} & 0 & $145.60 \pm 1.33$ & $146.20 \pm 1.24$ & $147.60 \pm 4.55$ & NS & $141.40 \pm 1.03$ & $141.80 \pm 0.86$ & $143.20 \pm 1.02$ & NS & $147.20 \pm 1.85$ & $146.60 \pm 1.60$ & $146.00 \pm 1.92$ & NS \\
\hline & 45 & $142.80 \pm 1.56$ & $148.40 \pm 1.33$ & $149.20 \pm 3.62$ & NS & $143.20 \pm 1.02$ & $144.80 \pm 0.86$ & $146.00 \pm 1.92$ & NS & $146.40 \pm 1.78$ & $151.00 \pm 2.74$ & $149.40 \pm 1.96$ & NS \\
\hline & 90 & $143.80 \pm 3.02$ & $151.40 \pm 1.03$ & $151.40 \pm 3.31$ & NS & $141.80 \pm 0.86$ & $148.60 \pm 1.86$ & $149.40 \pm 1.96$ & NS & $148.00 \pm 1.82$ & $153.20 \pm 2.20$ & $151.00 \pm 2.74$ & NS \\
\hline
\end{tabular}

Superscripts are read row wise for comparison of means. Means in the same row with different superscript $a$, $b$, are significantly different $\left(p^{*}<0.05\right)$ NS= Non-significant.

\section{Milk Production}

The milk yield of different groups of cows at different the agro-climatic zones has been presented in the [Table-6]. In the experimental duration there was no significant difference in the milk yield of cows due to either commercial or area specific mineral supplementation from that of un-supplemented group at Hill and Plain zones. However, significantly $(p<0.05)$ higher milk yield during $60^{\text {th }}$ and $90^{\text {th }}$ days was recorded in control and treatment groups than negative control groups at plateau zone. Panda et al.,2016 [9] observed that serum concentration of $\mathrm{Ca}, \mathrm{P}$ and some trace minerals were not changed during early period of supplementation whereas the same minerals were changed during the later days of supplementation, might be due to better bioavailability of minerals and better homeostasis by the cows when adopted. Singh et al., 2008 [9] found that milk production percentage increased as phosphorous increased from 0.03 to 0.42 percent compared with 0.24 percent in lactating cattle. Such effects might have caused a significant difference in milk yield towards later part of experiment.

Table-6 Mean value of Milk production ( $\mathrm{ml})$ in cow supplemented either area specific (T) or commercial mineral mixture (C) in different agro-climatic zones

\begin{tabular}{|c|c|c|c|c|c|c|c|c|c|c|c|c|}
\hline \multirow[t]{2}{*}{ Days } & \multicolumn{3}{|c|}{ Hill } & \multirow[t]{2}{*}{ Sig. } & \multicolumn{3}{|c|}{ Plateau } & \multirow[t]{2}{*}{ Sig. } & \multicolumn{3}{|c|}{ Plain } & \multirow[t]{2}{*}{ Sig. } \\
\hline & NC & C & $\mathrm{T}$ & & NC & C & $\mathrm{T}$ & & NC & C & $\mathrm{T}$ & \\
\hline 0 & $\begin{array}{r}502.00 \\
\pm 41.88\end{array}$ & $\begin{array}{r}614.00 \\
\pm 64.62\end{array}$ & $\begin{array}{r}574.00 \\
\pm 49.25\end{array}$ & NS & $\begin{array}{r}506.00 \\
\pm 42.26\end{array}$ & $\begin{array}{l}576.00 \\
\pm 49.05\end{array}$ & $\begin{array}{r}566.00 \\
\pm 50.85\end{array}$ & NS & $\begin{array}{r}606.00 \\
\pm 63.84\end{array}$ & $\begin{array}{l}758.00 \\
\pm 99.82\end{array}$ & $\begin{array}{l}780.00 \\
\pm 99.45\end{array}$ & NS \\
\hline 30 & $\begin{array}{r}526.00 \\
\pm 40.82\end{array}$ & $\begin{array}{r}634.00 \\
\pm 66.53 \\
\end{array}$ & $\begin{array}{r}590.00 \\
\pm 52.63\end{array}$ & NS & $\begin{array}{r}532.00 \\
\pm 39.17 \\
\end{array}$ & $\begin{array}{r}640.00 \\
\pm 40.62\end{array}$ & $\begin{array}{r}624.00 \\
\pm 41.79\end{array}$ & NS & $\begin{array}{r}636.00 \\
\pm 74.00 \\
\end{array}$ & $\begin{array}{c}770.00 \\
\pm 101.93\end{array}$ & $\begin{array}{c}806.00 \\
\pm 110.80\end{array}$ & NS \\
\hline 60 & $\begin{array}{r}546.00 \\
\pm 40.20\end{array}$ & $\begin{array}{r}664.00 \\
\pm 60.13 \\
\end{array}$ & $\begin{array}{r}618.00 \\
+55.98 \\
\end{array}$ & NS & $\begin{array}{r}556.00^{b} \\
\pm 41.67\end{array}$ & $\begin{array}{l}690.00^{a} \\
\pm 32.71\end{array}$ & $\begin{array}{r}674.00 \mathrm{a} \\
\pm 39.82 \\
\end{array}$ & * & $\begin{array}{r}610.00 \\
\pm 65.12 \\
\end{array}$ & $\begin{array}{r}794.00 \\
\pm 105.86\end{array}$ & $\begin{array}{c}854.00 \\
\pm 113.56\end{array}$ & NS \\
\hline 90 & $\begin{array}{r}542.00 \\
\pm 36.52 \\
\end{array}$ & $\begin{array}{r}708.00 \\
\pm 62.32 \\
\end{array}$ & $\begin{array}{r}650.00 \\
\pm 54.95\end{array}$ & NS & $\begin{array}{l}558.00^{\mathrm{b}} \\
\pm 41.52 \\
\end{array}$ & $\begin{array}{l}766.00^{\mathrm{a}} \\
\pm 35.30\end{array}$ & $\begin{array}{r}764.00 \mathrm{a} \\
\pm 35.86 \\
\end{array}$ & * & $\begin{array}{r}636.00 \\
\pm 68.96 \\
\end{array}$ & $\begin{array}{r}824.00 \\
\pm 106.99\end{array}$ & $\begin{array}{c}892.00 \\
\pm 112.71\end{array}$ & NS \\
\hline
\end{tabular}

Means in the same row with different superscript $a, b$, are significantly different $\left({ }^{*} p<0.05\right)$ NS= Non-significant

\section{Conclusions}

It may be concluded that specific required mineral if supplemented judiciously along with regular feeds would result in normal serum biochemical profile as that of commercially available mineral mixture. More extensive study regarding effects of area specific mineral mixtures compared to commercial mineral mixtures on serum biochemical parameters and milk mineral profile need to be done so as to adopt area specific mineral supplementation in cattle feeding.

Application of research: Research is applicable for dairy farmers and extension worker of the state for minimizing unnecessary use of mineral and for 
the economic dairy farming.

Research Category: This is research study for the assessment to use specific mineral as the availability of mineral in the different agro-climatic condition of state.

Author Contributions: All author equally contributed

Author statement: All authors read, agree and approved the final manuscript

Conflict of Interest: None

Ethical approval: This is the research work of M.V.Sc. student and approval has been taken from ethical committee constituted by Veterinary Council of India, New Delhi.

Acknowledgement: Authors are highly thankful to the Chhattisgarh Kamdhenu Vishwavidyalaya, Raipur, Chhattisgarh 492006 and concerned Veterinary Assistant Surgeon of study area District of Chhattisgarh for providing facilities and help to conduct study.

\section{References}

[1] Comerford, N.B. (2005) Soil Factors Affecting Nutrient Bioavailability. H Bassiri Rad Ed. Nutrient Acquisition by Plants an Ecological Perspective. Volume 181 Springer-Verlag Berlin Heidelberg ISBN: 978-3-540-24186-7 P-348.

[2] Bhausaheb K.S., Tiwari S.P., Sahu T., Naik S.K. and Gendley M.K. (2014) International Journal of Advanced Research, 2, 1076-1082.

[3] Griffiths L.M., Loeffler S.H., Socha M.T., Tomlinson D.J. and Johnson A.B. (2007) Animal Feed Science and Technology, 137, 69-83.

[4] Gopinath D., Sharma M.C., Gurupriya V.S. and Singh M. (2015) India International Journal of Agriculture Science and Veterinary Medicine, (2) 3 , 2320-3730.

[5] Snedecor G.W. and Cochran W.G. (1994) Statistical Methods. 8th Edition. lowa State University Press, Ames, lowa

[6] Chaudhary R.K, Patel D.C. and Parnerkar S. (2015) Life Science Leaflets. 62, 28-35.

[7] Sharma J., Kumar A., Tiwari D.P. and Mondal B.C. (2011) Indian Journal of Animal Science, 81 (5), 493-97.

[8] Doornenbal H., Tong A.K.W. and Murray N.L. (1988) Canadian Journal of Veterinary Research, 52, 99-105.

[9] Exton J.H. (1980) American Journal of Physiology, 3, 235-238.

[10] Kumar P., Sharma M.C. and Joshi C. (2005) Indian Journal of Animal Science, 75(8), 909-914.

[11] Panda M., Panda N., Panigrahi B., Swain R.K. and Mishra A. (2016) Indian Journal of Animal Nutrition, 33 (3), 290-295.

[12] Singh V.K., Singh P., Verma A.K. and Mehra U.R. (2008) Livestock Research for Rural Development, 20 (8), Article 130. http://www/rrdorg//rrd20/8/singh20130htm 